\title{
Student t-test for Scalable Topology for TSCH based IIoT Networks
}

\author{
Seema Kharb ${ }^{1 *}$ and Anita Singhrova ${ }^{2}$ \\ ${ }^{1,2}$ Computer Science \& Engineering Department, DCRUST, Murthal, India \\ 1*seema016.phd@gmail.com, ${ }^{2}$ nidhianita@gmail.com
}

\begin{abstract}
The Industrial Internet of Thing (IIoT) is a logical extension of automation and connectivity, that has evolved as an integral part of an industrial domain known as machine to machine communication. The IIoT networks have stringent requirements of traffic differentiation, reliability, low power consumption, scalability and timeliness for its various applications. Time Slotted Channel Hopping (TSCH) is the technology that provides ultralow power and reliable communication to IIoT networks. Since, IIoT involves large number of devices, thus scalability of the network is the key concern nowadays. Accordingly, a scalable topology is to be selected for TSCH based IIoT networks. This work identifies the major topologies being used in industrial networks. Based on their limitations and key features, a two-tier topology is proposed for handling traffic differentiation and scalability in TSCH environment. The simulations for linear, star, mesh and proposed two-tier is carried out using 6TiSCH simulator. The proposed two-tier showed better results for large number of connected nodes and transmission cells, i.e., dense networks. The results were further verified by the method of hypothesis testing.
\end{abstract}

Keywords: Student's t-test, 6TiSCH Simulator, transmission cells, number of connected nodes

\section{Introduction}

Industrial Internet of Things (IIoT) is a system that connects machines, controllers, sensors etc., to provide a foundation for smart homes, smart cities, smart manufacturing and smart factories. These smart factories will incorporate intelligent monitoring in traditional factories and make them adaptable and efficient. For this purpose, several standards based on IEEE 802.15.4 like WirelessHart, ISA100.11a, Zigbee etc., have been designed. The unsuitability of IEEE 802.15.4 for Industrial networks has led to the foundation of IEEE 802.15.4e standard in 2012 [1], with various MAC layer amendments .Time Slotted Channel Hopping (TSCH) is one of those MAC layer amendments that supports application domains of process automation, control and equipment monitoring. Moreover, through multichannel communication and channel hopping it is able to provide low power and reliable communication. Therefore, it becomes a candidate solution for Industrial Internet of Things (IIoT).

An efficient IIoT system must be able to meet the stringent requirements of power consumption, reliability, latency, service differentiation, interoperability, scalability and efficient selection of network topology [2][5]. Network topology has a significant impact on various network performance metrics like energy consumption, latency, QoS etc. Further, network topology plays an important role in designing an efficient scalable network. The details for some of the existing projects in IWSN and the topologies used in them has been discussed in Table 1. After the detailed review, it has been observed that

Received (May 6, 2018), Review Result (July 24, 2018), Accepted (August 8, 2018)

* Corresponding Author 
based on the application and size of the network different topologies are considered for deployment. Thus, the topology has a noticeable impact on the performance of the network.

Table 1. A Comparison of Existing IWNs Projects Adopted from [4]

\begin{tabular}{|l|l|l|l|l|}
\hline Study & Topology & Protocol & Medium & Target application \\
\hline Zhang et al. [6] & Mesh & ZigBee & $\begin{array}{l}\text { Wired and } \\
\text { wireless }\end{array}$ & Underground coal mine \\
\hline Fontana et al. [7] & Star & N/A & Wireless & Pollution of HV insulators \\
\hline Yoon et al. [8] & Mesh & Wi-Fi & Wireless & Monitoring oil pipe \\
\hline Hou and Bergmann [9] & Star & ZigBee & $\begin{array}{l}\text { Wired and } \\
\text { wireless }\end{array}$ & Monitoring machine \\
\hline $\begin{array}{l}\text { Automation System Co., } \\
\text { Ltd [10] }\end{array}$ & Star & N/A & Wireless & $\begin{array}{l}\text { Automation control of } \\
\text { cement kiln }\end{array}$ \\
\hline Company of MOXA [11] & Star & Wi-Fi & Wireless & Organization of warehouse \\
\hline $\begin{array}{l}\text { Bayindir and Cetinceviz } \\
{[12]}\end{array}$ & Star & Wi-Fi & $\begin{array}{l}\text { Wired and } \\
\text { wireless }\end{array}$ & Water pumping control \\
\hline
\end{tabular}

The network topologies majorly used are star, linear and mesh, [5]. In the star topology, all nodes are connected directly to a central entity known as root or sink node. Although, it provides lower latency, but results in scalability issues in larger networks. In the fully mesh topology, each node is connected to rest of the nodes in the network. The aforesaid topology is also known as flat or unstructured topology and results in a self-healing network having high reliability. On the other hand, it causes flooding of redundant messages in the network, which leads to the wastage of the bandwidth, increased latency, and reduced network life. Also, in the case of linear topology, the delay and topology maintenance overhead are very high.

After assessing these discussions and real-time applications [13], it was observed that the variant of these topologies is more trueful representation in an industrial network. The reason for this is that in an industry or production plant the complete process is divided into several zones based on their functionality where the nodes belonging to the same category are kept in the same zone. The nodes of one zone communicate to each other more frequently than with the nodes of another zone. Therefore, two-tier topologies are generally used in industries like a line - star and star-star. Therefore, the objective of this paper is to determine the scalable behavior of most commonly used topologies for TSCH based IIoT networks. The main topologies used in TSCH are discussed and a two-tier topology is proposed for handling the major requirements of industrial systems, i.e., traffic differentiation and scalability. In this architecture sensors are divided into clusters based on their functionalities. Here, the cluster heads directly communicate to the central coordinator and forms a star topology. Therefore, the major contributions of this article are: to design a two-tier topology, to implement star, mesh, linear and two-tier topology and then analyzing the scalability behavior of different topologies in TSCH environment through simulations and hypothesis testing.

The rest of the paper is organized as follows. Section 2 discusses basic features of TSCH. Section 3 describes the proposed 2tier architecture. Section 4 presents the simulative assessment of scalability of topologies. The hypothesis testing to evaluate the scalable behavior of topologies in TSCH environment is presented in Section 5, followed by discussions of results in Section 6. Finally, the conclusion and future work are listed in Section 7. 


\section{Time Slotted Channel Hopping (TSCH)}

Time slotted channel Hoping (TSCH) combines the benefits of slotted communication and channel hopping with multichannel capabilities to provide deterministic and reliable behavior to industrial network. This section discusses basic features of TSCH.

Slot-frame: In TSCH, the time is sliced into time-slots of equal duration. These timeslots are grouped together to form a slot-frame which repeats over time. Each slot is identified by a unique id known as Absolute Slot Number (ASN). There can be a single or multi slotframe within the network. Figure 1 shows an example of slot-frame with 4 slots and 6 channel offsets.

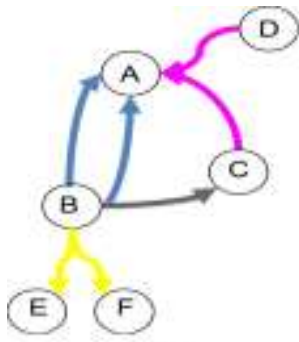

(a)

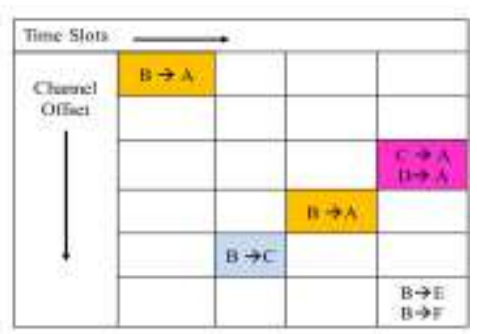

(b)

\section{Figure 1. An Example of (a) Industrial Network (b) Slot-frame}

Scheduling Algorithm: In a slot-frame, slots are allocated to nodes for communication. The procedure that performs this functionality is known as the scheduling algorithm. A scheduling algorithm can be either centralized or distributed in nature. In a centralized algorithm, a central entity is responsible for creating and distributing the schedule. While in a distributed algorithm, nodes create a schedule on the basis of their local information. A number of centralized and distributed algorithms are discussed in the literature [15]-[24].

Channel Hopping: In order to mitigate the effect of interference and multipath fading channel hopping is used. The TSCH can support 16 channels on $2.4 \mathrm{GHz}$ ISM band, where each channel is identified through a channel offset $\left(\mathrm{Ch}_{\mathrm{of}}\right)$. The mapping $(\mathrm{F})$ of channel offset to a frequency (f) is done using equation (1).

$$
f=F\left[\left(A S N+C h_{o f}\right) \bmod n_{c h}\right]
$$

where, $\mathrm{n}_{\mathrm{ch}}$ is number of channels available.

\section{Proposed two-tier Network}

This section presents the assumptions and algorithm for creating the proposed two-tier network. Considering that a network consists of ' $n$ ' number of nodes where there is only one sink node and rest of the ' $n-1$ ' nodes are distributed into ' $\mathrm{m}$ ' clusters. Each cluster represents a distinct process zone and has an associated cluster head. The cluster heads gather the data from nodes in their respective cluster. The nodes can also communicate to each other within the cluster. Thus, forming a mesh network with cluster head as the sink node at the second tier. The cluster heads are single hop away from the sink node and form a star topology network with sink node at first tier. To scale an industrial network two-tier topology can be deployed so as to aggregates multiple smaller networks.

\subsection{Assumptions}

Following assumptions are made: 
i. All nodes are deployed randomly to monitor and control the environment.

ii. Heterogeneous networks are considered supporting applications with different requirements like safety, control, and monitoring.

iii. Initially, all nodes are at the same energy level.

iv. A node will be in one of the three roles, i.e., sink(root) node, cluster head or cluster member.

v. All sensor nodes except the root belong to any one of the cluster.

vi. A node id and a zone_id is associated with each node.

vii. The distance between two nodes is computed as a function of reduced signal strength indicator (RSSI).

\subsection{Proposed Algorithm}

Each node is identified by a unique identifier 'id'. Here, six different zones ( 0 to 5$)$ that supports three broad categories of industrial applications, i.e., safety, control and monitoring [14] are considered.

This algorithm is repeated for each node. The first node, having id 0 is set as the root node. The root node does not belong to any of the cluster so its zone_id is set to be -1. For nodes other than the root node, the zone_id is randomly assigned from 0 to 5 . The first node in each zone will act as a cluster head. After that, the deployment routine places root node in the center of the area and cluster heads at a single hop distance from it, forming a star type topology. The step wise details for creating two-tier architecture is given in Algorithm1.

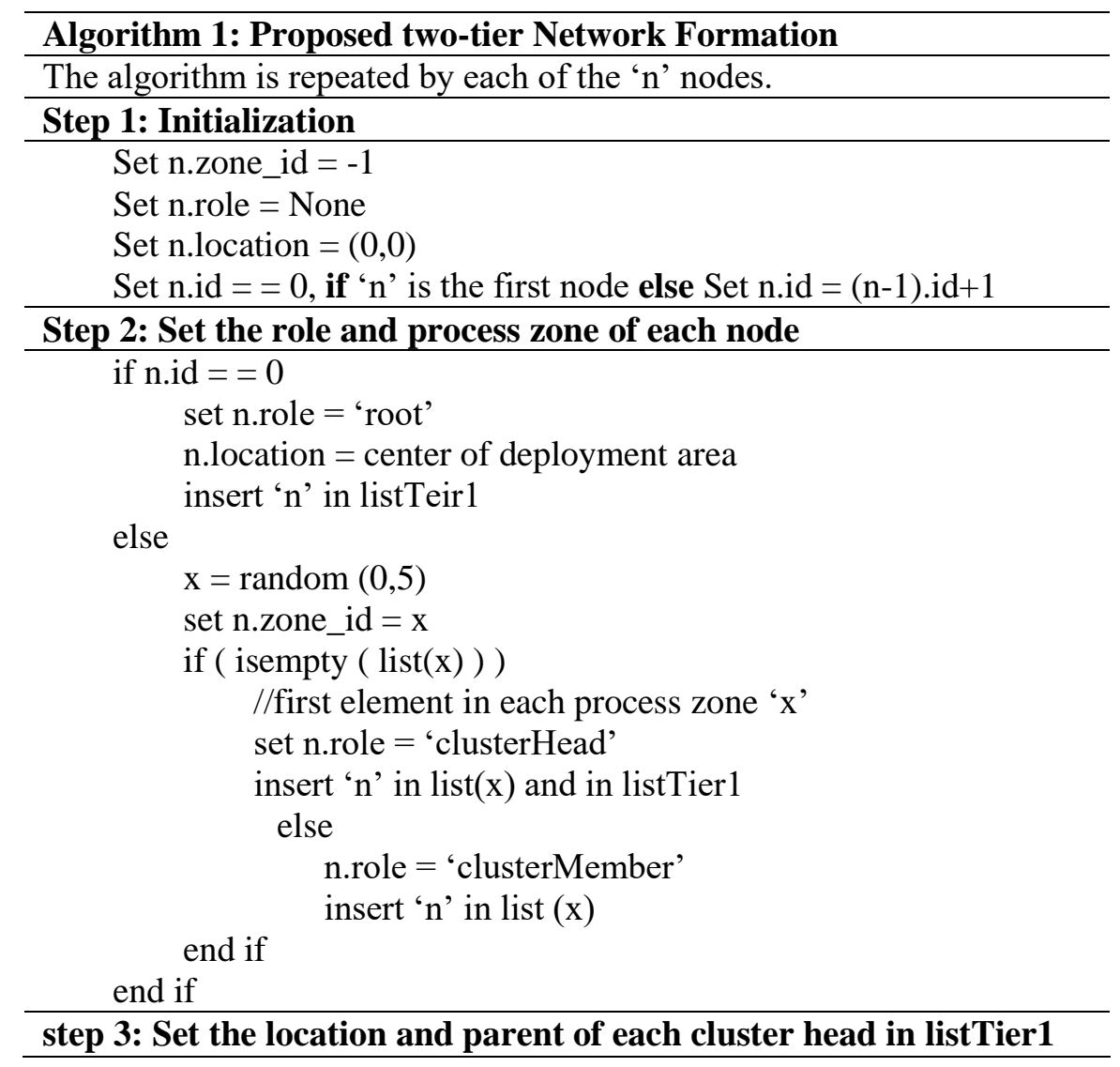




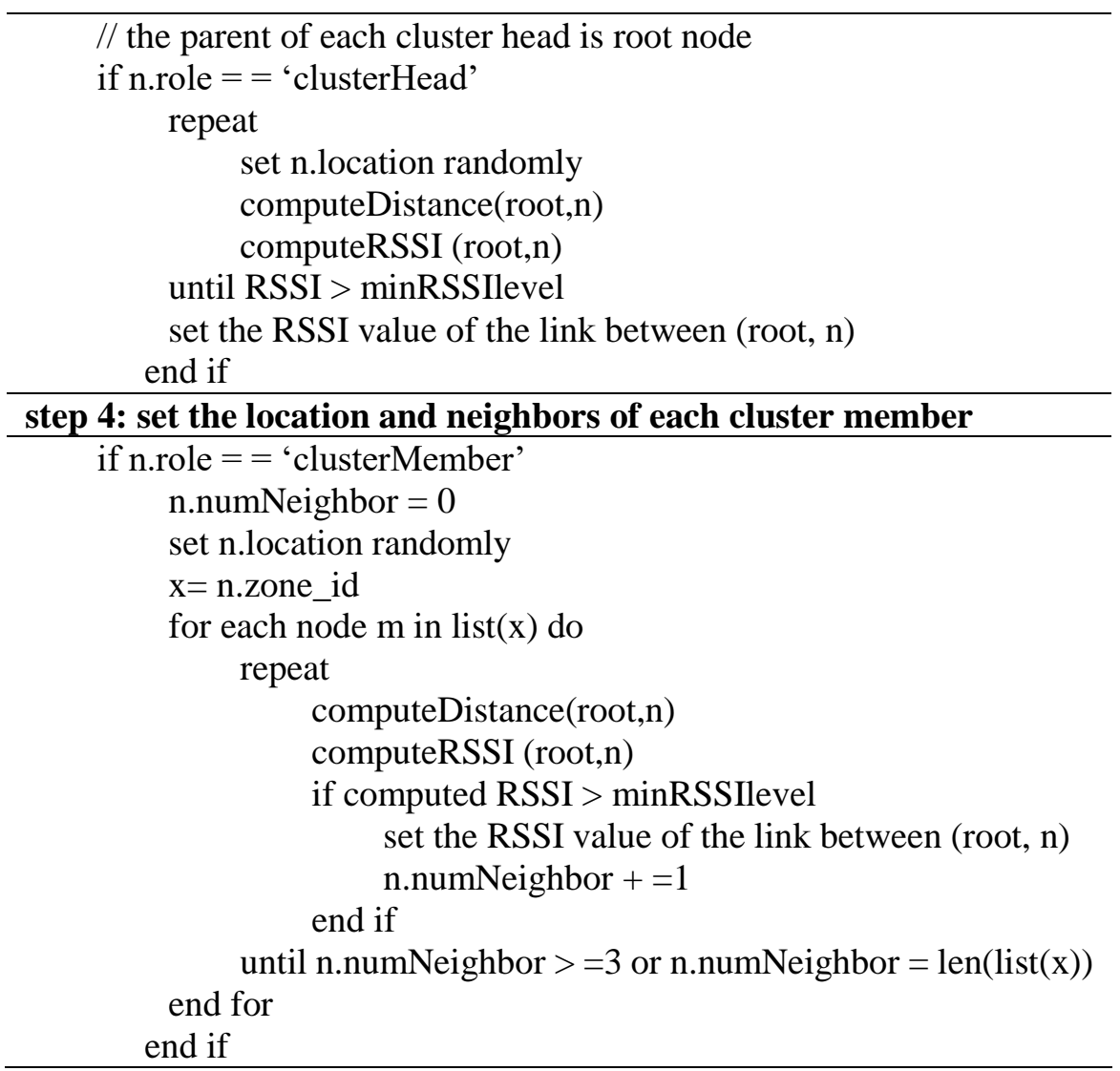

\section{Hypothesis Testing}

Hypothesis testing is a procedure that is used to accept or reject a hypothesis. There are different methods for hypothesis testing like t-test, f-test, z-test. In this work, t-test is used due to its robustness against assumptions. This section firstly describes the general procedure for applying a t-test and then the hypothesis is defined to test the scalability of each topology in TSCH environment, followed by the results of hypothesis testing.

\subsection{General Procedure for the t-test}

The complete procedure for t-test is explained in the steps given below.

Step 1: Assumption Validation. The major assumptions are related to the normality of data. There are three main assumptions. The data should be continuous. There should be no outliers and the data should be normally distributed. There are different methods through which the test of normality and outliers are performed. In this work, histograms and boxplots are used for checking normality and outliers respectively.

Step 2: State the Null Hypothesis $\left(H_{0}\right)$. It generally represents that there is no significant difference between samples. According to R.A. Fisher, "Null hypothesis is the hypothesis which is tested for possible rejection under the assumption that it is true."

Step 3: State the Alternate Hypothesis $\left(H_{a}\right)$. It represents that there is a significant difference between the samples and is complementary to the null hypothesis. It can take three forms.

$$
H_{a}:\left\{\begin{array}{cc}
\mu \neq \mu_{0} & (\text { two }- \text { tailed }) \\
\mu>\mu_{0} & (\text { right }- \text { tailed }) \\
\mu<\mu_{0} & (\text { left tailed })
\end{array}\right.
$$


Step 4: Level of Significance $(\alpha)$. It represents the probability that a random variable will belongs to the critical region. Generally, its value is $5 \%$ or $1 \%$. In this work, $5 \%$ level of significance is used.

Step 5: Test Statistics $(t)$. It computes the test statistics under the null hypothesis using the formula,

$$
t=\frac{\bar{\mu}}{S . E(\bar{\mu})}
$$

where, $\bar{\mu}=$ mean of the difference between two sample and $\mathrm{S} . \mathrm{E}(\bar{\mu})$, is the standard error of mean difference and is given as,

$$
S . E\left(\overline{\mu)}=\frac{\text { standard deviation of difference }}{\sqrt{n}}\right.
$$

Step 6: Make Decision. The computed t-value is compared with the tabulated value (pvalue) at significance level and decision related to acceptance or rejection of null hypothesis is taken.

\subsection{Student's t-test for Linear Topology}

In case of linear networks, a chain of nodes is formed, where one node is linked with the previous node. In case of TSCH, RPL routing protocol is used, which associates a rank with each node. In an idle case, all nodes are linked and have an associated rank value. So, the mean difference between the number of connected nodes and the number of nodes in a scalable network is zero. Hence, the proposed null $\left(\mathrm{H}_{0}\right)$ and alternate $\left(\mathrm{H}_{\mathrm{a}}\right)$ hypothesis are: -

$$
\begin{gathered}
\mathrm{H}_{\mathrm{L} 0}: \boldsymbol{\mu}=0, \text { the network is scalable } \\
\mathrm{H}_{\mathrm{La}}: \boldsymbol{\mu} \neq 0, \text { the network is not scalable. }
\end{gathered}
$$

\subsection{Student's t-test for Star Topology}

To evaluate the scalability of a star network, the number of transmission cells allocated is used as one of the parameter. In an idle case, the minimum number of transmission cell allocated to each node is one, as each node is connected to root node only. So, the difference between the means of the number of nodes and transmission cells is 1 . Hence, the following hypothesis is proposed.

$$
\begin{gathered}
\mathrm{H}_{\mathrm{s} 0}: \boldsymbol{\mu}=1, \text { the network is scalable, } \\
\mathrm{H}_{\mathrm{S} a}: \boldsymbol{\mu} \neq 1 \text {, the network is not scalable. }
\end{gathered}
$$

\subsection{Student's t-test for Mesh and Proposed 2-tier}

In case of mesh and proposed 2-tier networks, each node can be linked with a maximum of three nodes (simulator assumption). In the worst case, each node is linked with three other nodes and each link has an associated transmission cell. So, the maximum number of transmission cells will be three times the number of nodes and the mean difference between the two samples will be two times the mean value of sample 1 (i.e., number of nodes). Let ' $\mathrm{D}$ ' be the maximum mean difference. The null $\left(\mathrm{H}_{0}\right)$ and alternate $\left(\mathrm{H}_{\mathrm{a}}\right)$ hypothesis are defined as: -

$$
\begin{gathered}
\mathrm{H}_{\mathrm{M} 0}, \mathrm{H}_{20}: \boldsymbol{\mu}=\mathrm{D} \text {, the network is not scalable. } \\
\mathrm{H}_{\mathrm{Ma}}, \mathrm{H}_{2 \mathrm{a}}: \boldsymbol{\mu}<\mathrm{D} \text {, the network is scalable. }
\end{gathered}
$$




\subsection{Results of Statistical Analysis}

The observed values of $\mathrm{t}$-stat, $\mathrm{t}$ (critical), $\mathrm{p}$-value and alpha values of $\mathrm{t}$-Test for all four topologies are summarized in Table 2. Here, t-stats represents the observed or calculated value of t-statistics, t-critical tells the boundary value of rejection region, $\mathrm{p}$-value is the tabulated value of t-test computed from t-table and alpha represents the level of significance.

Table 2. Student's t-Test Statistics

\begin{tabular}{|l|r|r|r|r|}
\hline Topology & t - stat & t - critical & p-value & alpha \\
\hline Linear & 13.789 & 1.981 & $<0.0001$ & 0.05 \\
\hline Star & 4.869 & 1.993 & $<0.0001$ & 0.05 \\
\hline Proposed 2tier & -9.660 & -1.761 & $<0.0001$ & 0.05 \\
\hline Mesh & -17.866 & -1.761 & $<0.0001$ & 0.05 \\
\hline
\end{tabular}

The computed p-value, which represents tabulated value in all the four topologies is lower than the absolute value of t-stat at significance level alpha $=0.05$. So, the null hypothesis $\mathrm{H}_{0}$ is rejected, and the alternative hypothesis $\mathrm{H}_{\mathrm{a}}$ is accepted.

These results can also be verified by looking at the distribution of t-values in the graphs of the t-test. The Figure 2 (a),(b) shows that the performed t-test for the linear and star topologies is two-tailed and the observed or calculated value of t-statistics lies outside the distribution, i.e., $\mathrm{H}_{\mathrm{La}}$ and $\mathrm{H}_{\mathrm{Sa}}$ are accepted which means the network is not scalable.

The $t$ statistics of $t$-test for mesh and proposed 2tier topologies is shown in Figure 2 (c), (d). It has been observed from these figures, that t-test performed is left tailed and the observed values of t-statistics lie in the rejection region. Therefore, $\mathrm{H}_{2 \mathrm{a}}$ and $\mathrm{H}_{\mathrm{Ma}}$ are accepted which means the networks are scalable.

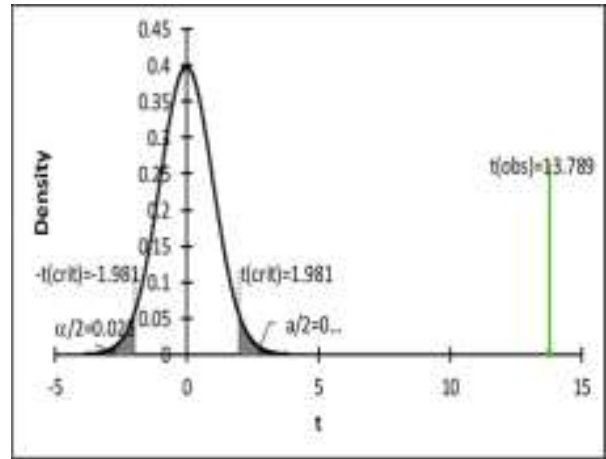

(a) Linear Network

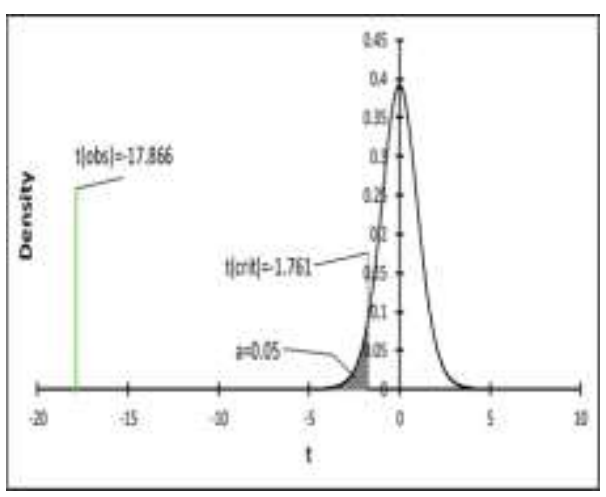

(c) Mesh Network

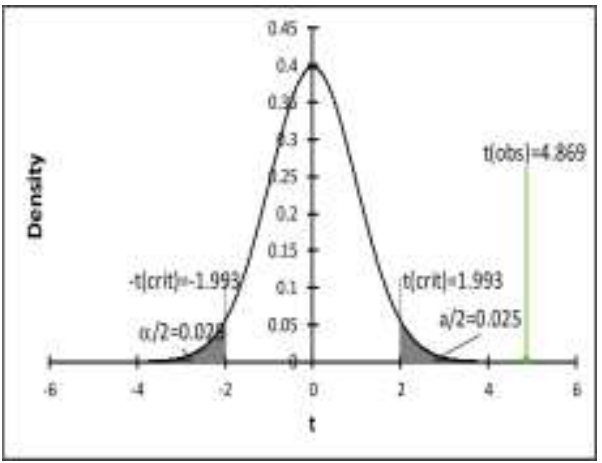

(b) Star Network

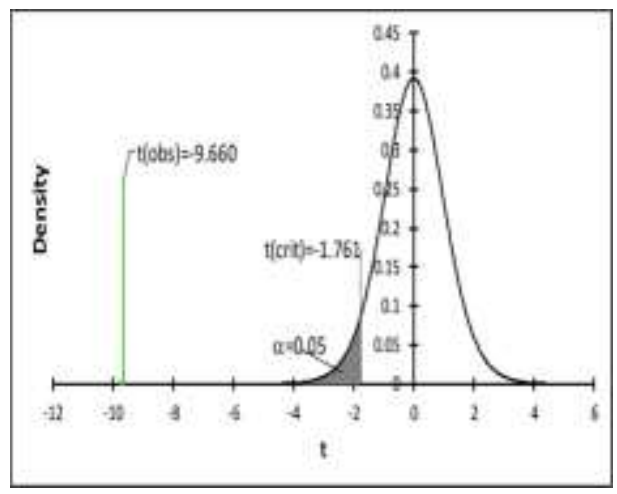

(d) Proposed 2tier Network

Figure 2. t-Test for Two Paired Samples for all Topologies 


\section{Simulative Assessment}

In order to evaluate the scalability of TSCH networks, four topologies namely, star, linear, mesh and proposed two-tier are considered. An example of these simulated topologies is shown in Figure 3. This section describes the simulation details like simulator used along with simulator settings, parameters used to evaluate the scalability performance and simulation results.

\subsection{Simulation Tool}

To study the scalability of TSCH, simulations are performed using 6TiSCH simulator [25]. It is an open source, event-based simulator, developed by the members of 6TiSCHWG and is written in Python. It models IEEE 802.15.4e-2012 TSCH at the MAC layer, routing protocol for LLNs (RPL) as the routing protocol and 6top [27] sublayer between $\mathrm{TSCH}$ and RPL for cell negotiations. Also, "Pister-Hack" propagation model with collisions is used as a PHY model, energy consumption model as specified in [28] and implements On The Fly (OTF) [24] as the scheduling algorithm.

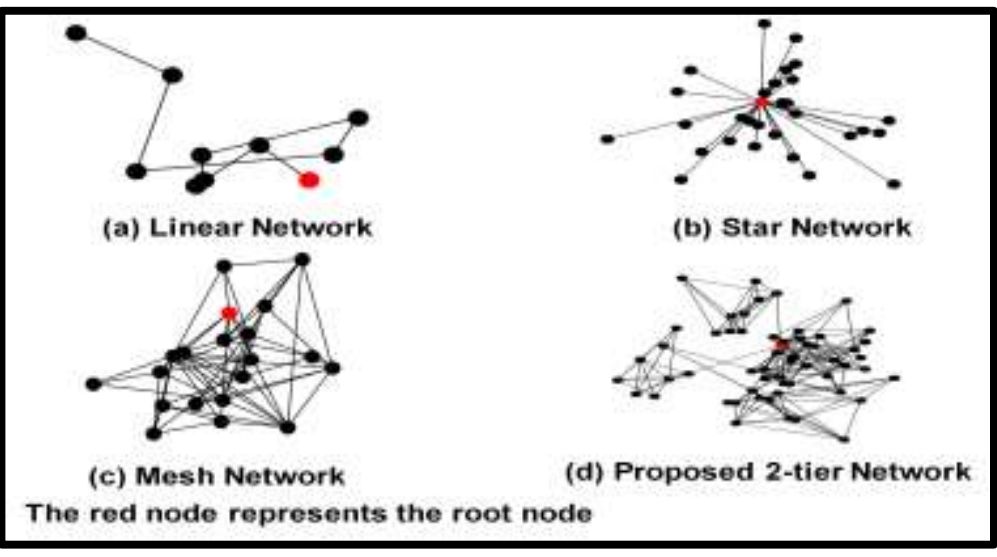

Figure 3. Example of Simulated Topologies

\subsection{Simulation Setup}

In order to evaluate the scalability limits of TSCH, networks with node density 10 to 1000 nodes are created. The nodes in the network are deployed randomly at random locations except for the root node which is deployed at the center of the deployment area. The simulation parameters corresponding to each pair of topology and number of nodes which are executed for five runs with 100 cycles each are shown in Table 3. For a single set, the results are average of 2000 values wherein a new topology is used for each run.

Table 3. Simulation Parameters

\begin{tabular}{|c|c|}
\hline \multicolumn{2}{|c|}{ Deployment /topology } \\
\hline Number of sensor nodes & 10 to 1000 \\
\hline Deployment area & $2 \mathrm{~km} \times 2 \mathrm{~km}$ \\
\hline Deployment constraint & $\begin{array}{l}3 \text { neighbors with PDR }>50 \% \text { and For star } 1 \\
\text { neighbor with PDR }>50 \%\end{array}$ \\
\hline Topology & [star, mesh, linear, proposed 2-tier] \\
\hline Application data generation period & $1 \pm 10 \%$ \\
\hline \multicolumn{2}{|r|}{ OTF } \\
\hline OTF threshold & $0,2,4,10$ slots \\
\hline OTF housekeeping period & $5 \mathrm{~s}$ \\
\hline
\end{tabular}




\begin{tabular}{|l|r|}
\hline Timeslot duration & $10 \mathrm{~ms}$ \\
\hline Slot-frame Length & 101 slots \\
\hline Number of Channels & 16 \\
\hline & RPL \\
\hline RPL_MIN_HOP_RANK_INCREASE & 256 \\
\hline MAX_ETX & 4 \\
\hline
\end{tabular}

\subsection{Performance Parameters}

A network is said to be scalable, if it is able to handle variable node density. Therefore, to determine the scalable behavior of $\mathrm{TSCH}$, number of connected nodes and number of allocated transmission cells are used as performance parameters.

Number of Connected Nodes (numConnectedNodes): Whenever a new node joins the network the routing protocol RPL assigns two rank fields to it, dagRank and rank [26][28]. It is a measure of the position of the node and is used by the neighbors to determine their relative positions w.r.t. each other. The dagRank of a node ' $n$ ' is a function of its rank and is computed as shown in eq. (4):

$$
\operatorname{dagRank}(n)=\frac{\operatorname{rank}(n)}{R P L_{-} M I N_{-} R A N K_{-} I N C}
$$

And the rank of a neighbor $(\mathrm{N})$ is computed through eq.(5):

$$
\operatorname{rank}(N)=\operatorname{rank}(\text { Parent })+\operatorname{rankIncrement}
$$

where, rankIncrement $=(3 * E T X-2) * R P L_{-} M I N \_R A N K_{-} I N C$

and ETX, is the Expected Transmission Count that represents the average number of packets transmitted between two nodes and RPL_MIN_RANK_INC is the constant set to a value of 256 . In order to identify the range of rank, following constants are also defined by the simulator,

$$
\begin{aligned}
& M A X_{-} R A N K_{-} I N C=2 * M A X_{-} E T X * R P L_{-} M I N_{-} R A N K_{-} I N C \\
& M A X_{-} T O T A L_{-} R A N K=256 * R P L_{-} M I \_R A N K_{-} I N C * 2
\end{aligned}
$$

If the rank of a node goes beyond the MAX_TOTAL_RANK its rank is set to "None". A node is said to be connected if it has an associated rank value. So, the numConnectedNodes represents the number of nodes having rank and is computes as given by eq. (9).

$$
\text { numConnectedNodes }=\operatorname{count}(\{n \mid \operatorname{rank}(n) !=\text { None }\})
$$

Number of Transmission Cells (numTransmissionCells), defines the total number of cells allocated by the scheduling algorithm to all the nodes to transmit their data. To compute this parameter, following information for each node is recorded, time-slot, neighbor-id, and slot-type(transmit/receive) and based on this information, number of Tx cells has been computed as in eq. (10),

numTransmissionCells $=\sum_{i=1}^{n} \operatorname{numTx}(i)+\sum_{i=1}^{n} \operatorname{numRx}(i)$

where, numTx and numRx represent the number of cells allocated to a node to transmit and receive the data respectively. 


\subsection{Simulation Results}

This subsection presents and discusses the simulation results for the performance parameters.

5.4.1. Number of Connected Nodes: The number of connected nodes is shown for linear, star, mesh and proposed two-tier network respectively as a function of node density in Figure 4(a), (b), (c), (d). It has been observed that except in case of linear topology the numConnectedNodes is equivalent to the number of nodes in rest of the topologies. This can be explained by considering the MAX_TOTAL_RANK constant that has a value of $131072(256 * 256 * 2)$ and as in linear each node can have maximum one child and one parent. So, each node ' $i$ ' can connect to node ' $\mathrm{i}-1$ ' resulting in a rank value higher than the parent node (from eq. (4)). By gathering the statistics of all nodes, it has been evaluated that in case of the linear, maximum value of rank reaches to around 192 194 nodes and no rank value is assigned to rest of the nodes. In case of star, all nodes are single hop away from the root node. So, the maximum rank increment is limited to rankIncrement only. On the other hand, in mesh and two-tier topologies each node can connect to a maximum of three nodes. So, if at any point a node is trying to link with a parent having maximum rank, a parent switch occurs.

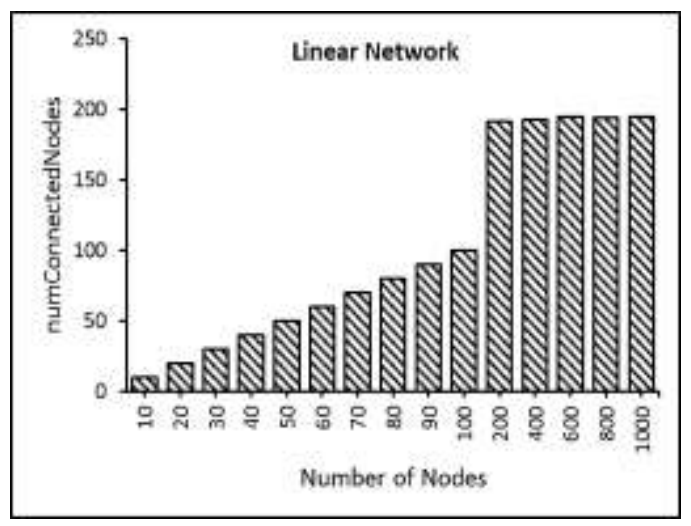

(a)

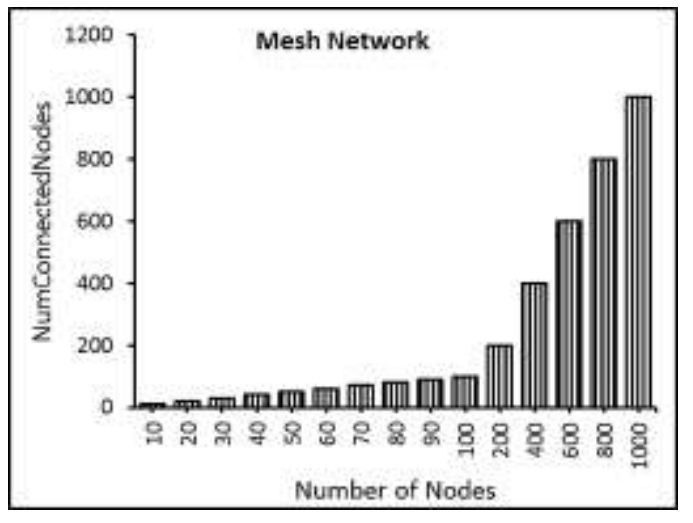

(c)

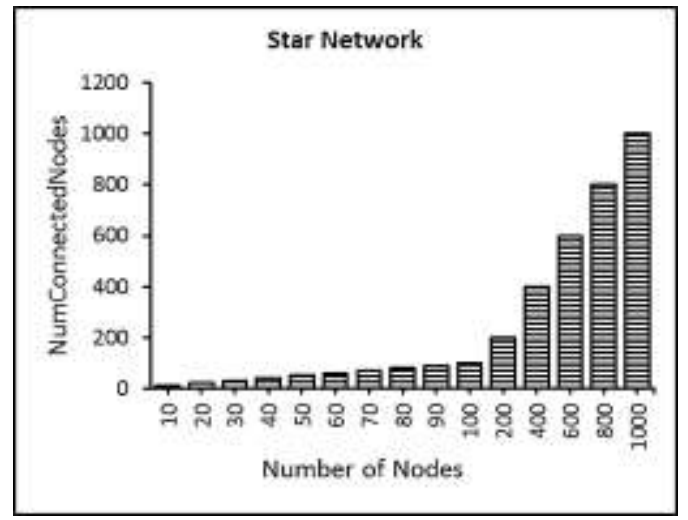

(b)

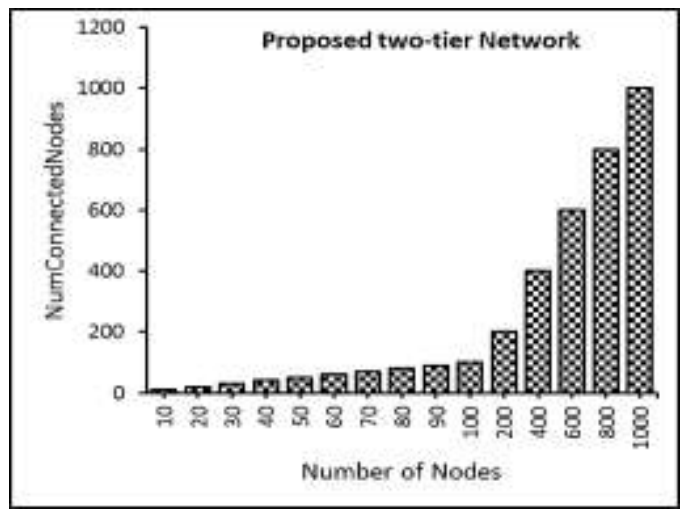

(d)

\section{Figure 4. Number of Connected Nodes in (a) Linear (b) Star (c) Mesh (d) Proposed 2tier}

5.4.2. Number of Transmission (Tx) Cells: The number of Tx cells allocated is depicted as a function of node density ,in case of different topologies in Figure 5(a), (b), (c), (d) respectively. It has been observed that in case of mesh and proposed 2tier, the number of transmission cells is increasing as the number of nodes increases. This can be explained as 
node density increases; more data packets will be generated and hence more number of transmission cells are required. But in case of linear and star topology, number of transmission cells became constant after a certain limit. On the other hand, in linear topology the number of connected nodes increases till 200 and after that it becomes steady, so is the number of transmission cells. In case of star topology too, the number of transmission cells increases up to a particular limit and then becomes steady. This variation is shown in Figure 6, where the number of transmission cells is plotted as a function of the number of nodes and slot-frame length. In a star topology, each node will communicate to the sink or root node only. So, there is no more than one time-slot allocated to each link. Hence, the bottleneck exists at the root. Even though multichannel communication is supported by TSCH, no two nodes can simultaneously transmit on same slot and different channel. Therefore, the maximum number of links to which a transmission cell is allocated, is restricted to slot-frame length $(51,101,151,201$, and 251).

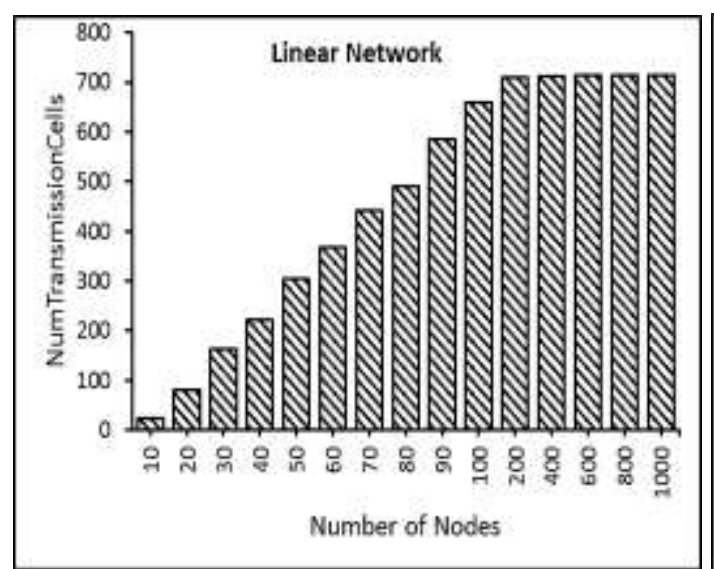

(a)

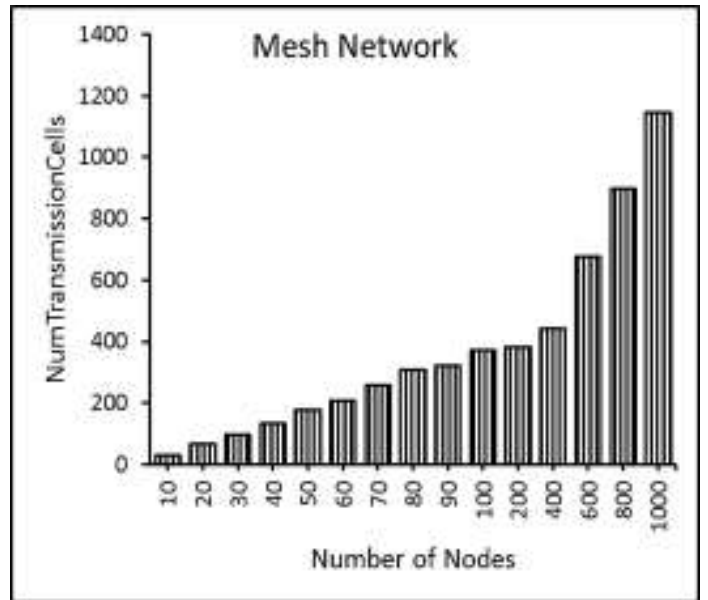

(c)

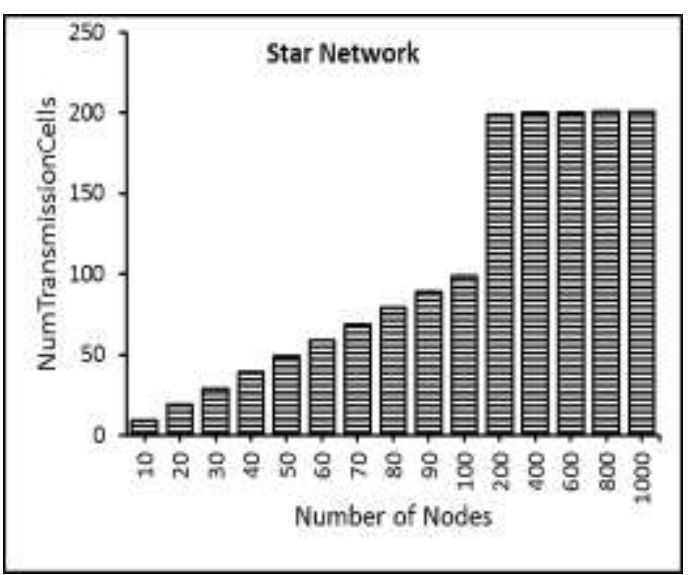

(b)

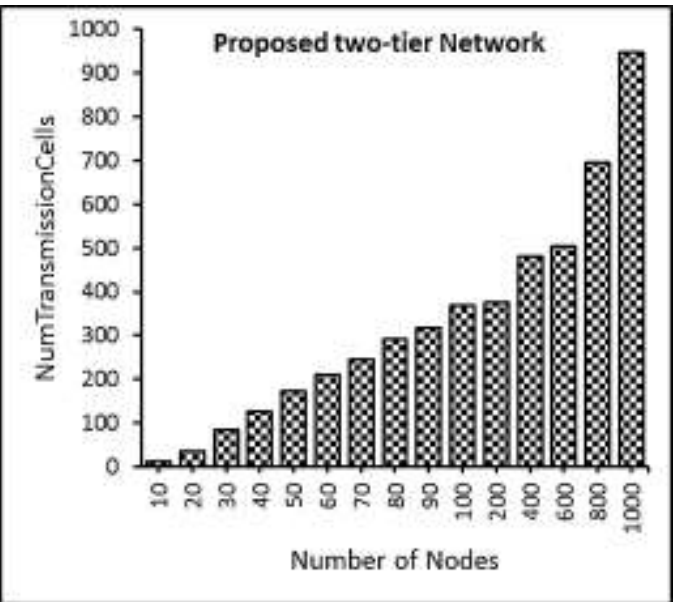

(d)

Figure 5. Number of Transmission Cells in (a) Linear (b) Star (c) Mesh (d) Proposed 2tier 


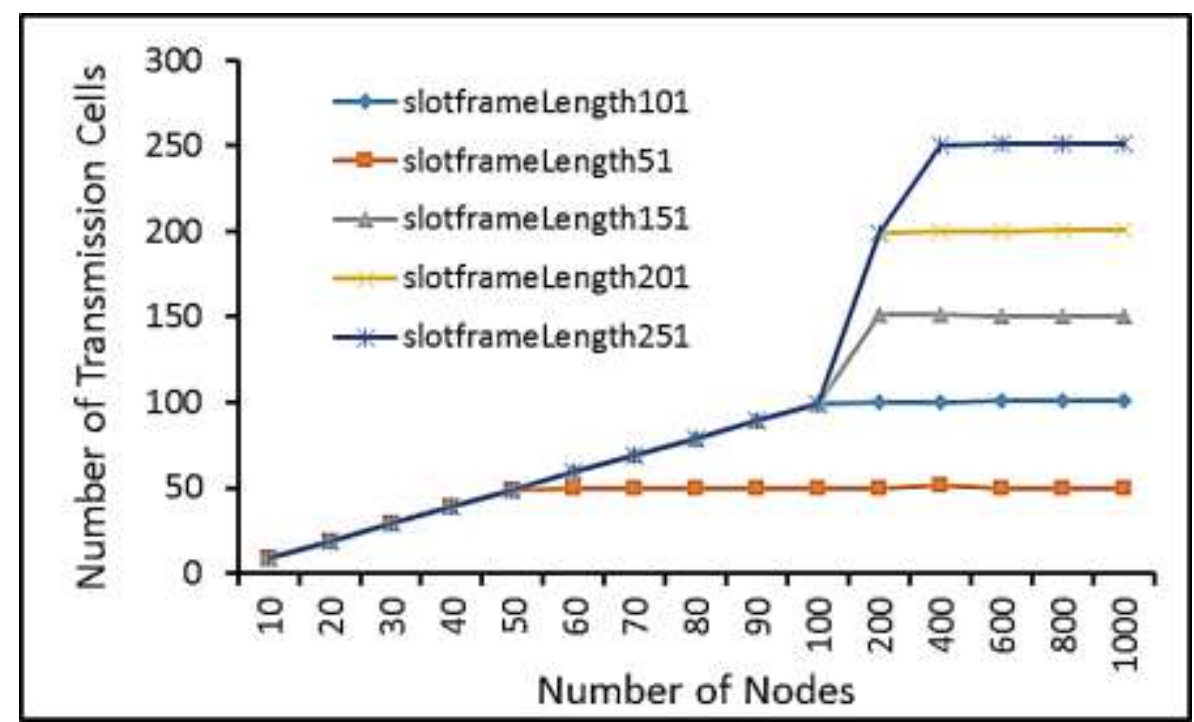

Figure 6. Number of Transmission Cells in Star Topology with different SlotFrame Length

5.4.3. Result Discussions: The simulation results show that star and linear networks reaches to a steady state after a particular node density and hence are not suitable for dense networks. This is in agreement with the results of [15] where star topology shows a decrease in reliability in the dense network. Also, two-tier provides less number of active cells per slot-frame than mesh networks. Hence, less energy consumption per node and improved battery or network life. On the other hand, the results of hypothesis testing signify that the observed t-value is less than the p-value, resulting in rejection of null hypothesis and acceptation of the alternate hypothesis. The alternate hypothesis in case of the star and linear states that the network is not scalable while in case of mesh and proposed 2tier, it states that network is scalable.

\section{Conclusion}

In this paper, a two-tier scalable network topology is proposed. The four topologies namely linear, star, mesh and two-tier are evaluated to identify their scalable behavior in TSCH based IIoT networks, using both simulative and statistical analysis. The results of hypothesis testing further compliments the simulation results, regarding the scalable behavior of topologies in TSCH environment. Hence, it can be concluded that mesh and proposed two-tier topologies show scalable behavior. Therefore, in case of a low-density network, any topology can be used based on the application requirements. But for high node density, only mesh and proposed two-tier are to be considered. As a future extension of this work, mesh and two-tier topologies will be further evaluated on other parameters like latency, collision handling and reliability etc.

\section{Acknowledgments}

This research has been supported by CSIR-Research Grants having grant number09/1063(0007)/2015-EMR-I.

\section{References}

[1] IEEE SA - 802.15.4e-2012 - IEEE Standard for Local and metropolitan area networks--Part 15.4: LowRate Wireless Personal Area Networks (LR-WPANs) Amendment 1: MAC sublayer, (2012), pp. 1-314. doi:10.1109/IEEESTD.2011.6012487. 
[2] P. McLaughlin and R. McAdam, "The Undiscovered Country: The Future of Industrial Automation", white paper, Honeywell. (2016).

[3] L. Da Xu, W. He and S. Li, "Internet of things in industries: A survey", IEEE Transactions on industrial informatics, vol. 10, no. 4, (2014), pp. 2233-2243.

[4] X. Li, D. Li, J. Wan, A. V. Vasilakos, C. F. Lai and S. Wang, "A review of industrial wireless networks in the context of industry 4.0", Wireless networks, vol. 23, no. 1, (2017), pp. 23-41.

[5] M. Raza, N. Aslam, H. Le-Minh, S. Hussain, Y. Cao and N. M. Khan, "A Critical Analysis of Research Potential, Challenges and Future Directives in Industrial Wireless Sensor Networks", IEEE Communications Surveys \& Tutorials, vol. 20, no. 1, (2017), pp. 39-95.

[6] Y. Zhang, W. Yang, D. S. Han and Y. I. Kim, "An integrated environment monitoring system for underground coal mines-wireless sensor network subsystem with multi-parameter monitoring", Sensors, vol. 14, no. 7, (2014), pp. 13149-13170.

[7] E. Fontana, J. F. Martins, S. C. Oliveira, F. J. M. M. Cavalcanti, R. A. Lima and G. O. Cavalcanti, "Sensor network for monitoring the state of pollution of high-voltage insulators via satellite", IEEE Transactions on Power Delivery, vol. 27, no. 2, (2012), pp. 953-962.

[8] S. Yoon, W. Ye, J. Heidemann, B. Littlefield and C. Shahabi, "SWATS: Wireless sensor networks for steamflood and waterflood pipeline monitoring", IEEE Network, vol. 25, no. 1, (2011), pp. 50-56.

[9] L. Hou and N. W. Bergmann, "Novel industrial wireless sensor networks for machine condition monitoring and fault diagnosis", IEEE Transactions on Instrumentation and Measurement, vol. 61, no. 11, (2012), pp. 2787-2789.

[10] Industrial wireless networks application in cement production. http://www.saiyuan.net/P/p154.html.

[11] Wireless roaming networking for automated guided vehicles. http://www.moxa.com/applications/Wireless_Roaming_Networking_for_Automated_Guided_Vehicles. htm.

[12] R. Bayindir and Y. Cetinceviz, "A water pumping control system with a programmable logic controller (PLC) and industrial wireless modules for industrial plants-An experimental setup", ISA Transactions, vol. 50, no. 2, (2011), pp. 321-328.

[13] M. F. Khan, E. A. Felemban, S. Qaisar and S. Ali, "Performance Analysis on Packet Delivery Ratio and End-to-End Delay of Different Network Topologies in Wireless Sensor Networks (WSNs)", IEEE Ninth International Conference on Mobile Adhoc and Sensor Networks (MSN), (2013), pp. 324-329.

[14] K. Pister, P. Thubert, S. Dwars and T. Phinney, "Industrial Routing Requirements in Low-Power and Lossy Networks", IETF Std. RFC5673, (2009).

[15] E. Municio and S. Latré, "Decentralized broadcast-based scheduling for dense multi-hop TSCH networks", In Proceedings of the Workshop on Mobility in the Evolving Internet Architecture, ACM, (2016), pp. 19-24.

[16] G. Alderisi, G. Patti, O. Mirabella and L. L Bello, "Simulative assessments of the IEEE 802.15. 4e dsme and tsch in realistic process automation scenarios", 2015 IEEE 13th International Conference on Industrial Informatics (INDIN), (2015), pp.948-955.

[17] S. Zats, R. Su, T. Watteyne and K. S. Pister, "Scalability of time synchronized wireless sensor networking", In IECON 2011-37th Annual Conference on IEEE Industrial Electronics Society, (2011), pp. 3011-3016.

[18] M. R. Palattella, N. Accettura, M. Dohler, L. A. Grieco and G. Boggia, "Traffic Aware Scheduling Algorithm for reliable low-power multi-hop IEEE 802.15. 4e networks", 2012 IEEE 23rd International Symposium on Personal Indoor and Mobile Radio Communications (PIMRC), (2012), pp. 327-332.

[19] R. Soua, P. Minet and E. Livolant, "MODESA: an optimized multichannel slot assignment for raw data convergecast in wireless sensor networks", 2012 IEEE 31st International Conference on Performance Computing and Communications (IPCCC), (2012), pp. 91-100.

[20] N. Accettura, E. Vogli, M. R. Palattella, L. A. Grieco, G. Boggia and M. Dohler, "Decentralized traffic aware scheduling in 6TiSCH networks: Design and experimental evaluation”, IEEE Internet of Things Journal, vol. 2, no. 6, (2015), pp. 455-470.

[21] A. Morell, X. Vilajosana, J. L. Vicario and T. Watteyne, "Label switching over IEEE802. 15.4 e networks", Transactions on Emerging Telecommunications Technologies, vol. 24, no. 5, (2013), pp. 458475.

[22] R. Soua, P. Minet and E. Livolant, "DiSCA: A distributed scheduling for convergecast in multichannel wireless sensor networks", 2015 IFIP/IEEE International Symposium on Integrated Network Management (IM), (2015), pp. 156-164.

[23] S. Duquennoy, B. Al Nahas, O. Landsiedel and T. Watteyne, "Orchestra: Robust mesh networks through autonomously scheduled tsch", In Proceedings of the 13th ACM conference on embedded networked sensor systems, (2015), pp. 337-350.

[24] M. R. Palattella, T. Watteyne, Q. Wang, K. Muraoka, N. Accettura, D. Dujovne and T. Engel, "On-thefly bandwidth reservation for 6TiSCH wireless industrial networks", IEEE Sensors Journal, vol. 16, no. 2, (2016), pp. 550-560.

[25] https://bitbucket.org/6tisch/simulator/.

[26] X. Vilajosana and K. Pister, "Minimal IPv6 over the TSCH Mode of IEEE 802.15.4e (6TiSCH) Configuration", RFC-8180, (2017) May. 
International Journal of Control and Automation

Vol. 11, No. 10 (2018)

[27] Q. Wang, X. Vilajosana and T. Watteyne, “6TiSCH Operation Sublayer (6top)”, IETF Std. draft-wang6tisch-6top-sublayer-11 [work-in-progress], (2018).

[28] X. Vilajosana, Q. Wang, F. Chraim, T. Watteyne, T. Chang and K. S. Pister, "A realistic energy consumption model for TSCH networks", IEEE Sensors Journal, vol. 14, no. 2, (2014), pp. 482-489.

[29] T. Winter, P. Thubert, A. Brandt, J. Hui, P. Levis, K. Pister, R. Struik, J. P. Vasseur and R. Alexander, "RPL: IPv6 Routing Protocol for Low-Power and Lossy Networks", RFC -6550, (2012). 